\title{
Elucidation of the SLP-65 phosphorylation state in activated B lymphocytes \\ T Oellerich ${ }^{* 1}$, M Gronborg'2, K Neumann¹, HH Hsiao², H Urlaub² and J Wienands ${ }^{1}$
}

\author{
Address: ${ }^{1}$ Georg-August University, Institute of Cellular and Molecular Immunology, Göttingen, Germany and ${ }^{2}$ Max Planck Institute for \\ Biophysical Chemistry, Bioanalytical Mass Spectrometry Group, Göttingen, Germany \\ * Corresponding author
}

from 12th Joint Meeting of the Signal Transduction Society (STS). Signal Transduction: Receptors, Mediators and Genes

Weimar, Germany. 29-31 October 2008

Published: 26 February 2009

Cell Communication and Signaling 2009, 7(SuppI I):A2I doi:I0.II86/I478-8IIX-7-SI-A2I

This abstract is available from: http://www.biosignaling.com/content/7/SI/A2I

(c) 2009 Oellerich et al; licensee BioMed Central Ltd.

The SH2 domain-containing leukocyte adaptor protein of $65 \mathrm{kDa}$ (SLP-65) is a central effector for signaling downstream of the B-cell antigen receptor (BCR). Upon phosphorylation on serine/threonine and tyrosine residues, SLP-65 nucleates the formation of multimolecular protein complexes to integrate numerous BCR-signaling events. We have now qualitatively and quantitatively identified phospho acceptor sites of SLP-65 by applying state of the art mass spectrometry. SLP-65 turned out to possess a plethora of phospho acceptor sites. In fact, it turned out to be one of the most phosphorylated proteins described so far. Moreover, by applying stable isotope labelling of amino acids in cell culture (SILAC) we identified several acceptor sites whose phosphorylation kinetic is differentially regulated upon BCR-stimulation. The functional relevance of some of these sites was subsequently analyzed by mutational analysis of SLP-65 in SLP-65-deficient DT40 B cells. In contrast to the described role of SLP-65 tyrosine phosphorylation for the initiation of $\mathrm{Ca}^{2+}$-signaling, serine/threonine phosphorylation of SLP-65 turned out to be a key regulator for BCR-dependent MAP-kinase activation and AP-1 regulated gene transcription. Collectively our data explain several of the SLP-65 controlled biological responses elucidated by genetic means and further support the role of SLP-65 as the key integrator of BCR-signaling. In general (phospho)proteomics combined with reconstitution experiments in gene-targeted DT40 cells proves to be a powerful strategy to uncover post translational modifications and their biological relevance in cell signaling. 